\title{
A Bio-inspired knowledge system for control tuning (BIO-KSY)
}

\author{
Jose Luis Calvo-Rolle, Ramón Ferreiro García \\ Departamento de Ingeniería Industrial \\ University of A Coruña \\ A Coruña, Spain \\ jlcalvo@udc.es
}

\author{
Emilio Corchado, Pedro Antonio Hernández, \\ María Luisa Pérez, María Araceli Sánchez \\ Departamento de Informática y Automática \\ University of Salamanca, Salamanca, Spain \\ escorchado@usal.es
}

\begin{abstract}
This study presents a novel bio-inspired knowledge system, based in closed loop tuning, for the calculation of the Proportional-Integral-Derivative (PID) controller parameters. The aim is to achieve automatically the best parameters according with the work point and the dynamics of the plant. For it, in our study, several typical expressions and systems have been taken into account to build the model. Each of these expressions is appropriated for a particular system. The novel method is empirically verified with a real dataset obtained by a liquid-level laboratory plant.
\end{abstract}

Keywords- industrial applications, bio-inspired models, artificial neural networks, knowledge-based system

\section{INTRODUCTION}

This study presents a novel method to build a simplified bioinspired model based on a closed-loop tuning for PID controller to calculate regulation parameters. Despite that the PID controller is one of the most traditional control type, researchers are still working with it to improve its behavior and performance [1-11]. There are several studies oriented to the same objective but always for specific systems [5, 9, 11].

Despite all this, there are a lot of controllers operating far from the most favorable state [14], overcoat controllers that are not of self-tuning type. Thus achieving new ways to solve this problem is indispensable. For many research works related with the PID controller, the method followed in many approaches is to try to obtain the best parameters according with the system. Another way is to achieve self tuning controller topologies [11, 12, 13].

The Bio-inspired Knowledge System (BIO-KSY) described in this study consists of two phases. The first phase is based on expert rules and the second one on a bio-inspired method. Both can achieve a BIO-KSY model with very good results. The basic element of the model based on rules only contemplates techniques with hard and very tested implantation in the industry. BIO-KSY is based in practical and robust methods; it is not a generic method that considers all the possibilities.

This paper is organized as follows. It starts with a brief description of the general diagram followed by the PID controller format explanation, and a concise review of PID controller tuning in a closed-loop. Afterwards BIO-KSY is exposed and finally an empirical verification is made; conclusions and future works are explained.

\section{THE GENERAL DIAGRAM TO STUDY THE PROPOSED BIO-KNOWLEDGE MODEL} 1.

The general schema of BIO-KSY is illustrated in figure

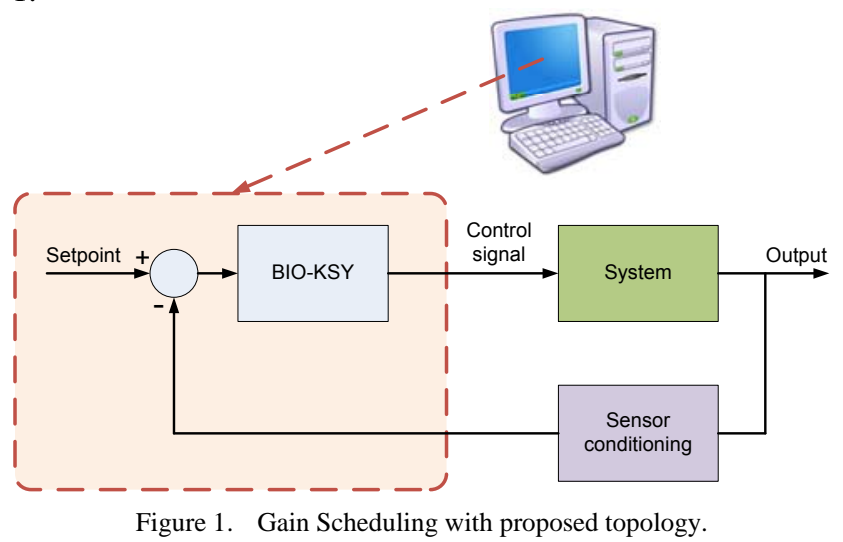

As it is shown in figure 1, the bio-inspired system is implemented in a computer. It is possible though to be tested through a data acquisition card; where it is possible to read the output and provide the control signal for the system. The set point is programmed on a computer. The Bio-Controller is based on traditional PID controller in standard format, and its tuning based on closed-loop method, specifically with relay-feedback technique, and hence it is applicable in many cases more than other methods. The obtained model to supply the controller with its parameters has an appearance similar to the one shown in figure 2 , where there is a part with rules and another one with artificial neural networks (ANN).

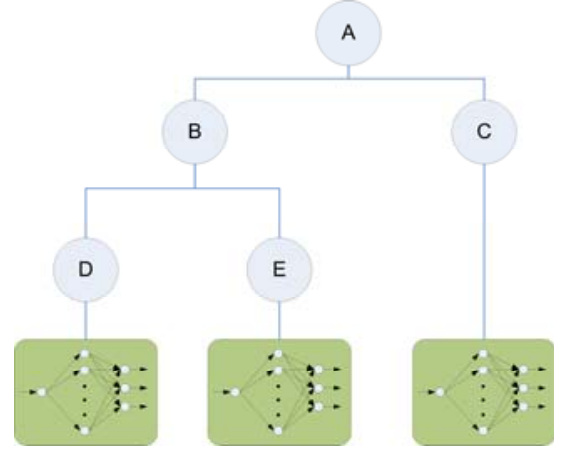

Figure 2. Model final structure. 


\section{PID CONTROLLER FORMAT}

There are several topologies of PID controllers, but in this study the standard format represented in equation 1 is employed $[14,15]$.

$$
u(t)=K\left[e(t)+\frac{1}{T_{i}} \int_{0}^{t} e(t) d t+T_{d} \frac{d e(t)}{d t}\right]
$$

Where ' $u$ ' is the control variable and ' $e$ ' is the control error given by ' $e=S P-y$ ' (difference between the setpoint ' $S P$ ' and conditioned output ' $y$ '). The other terms are the tuning controller parameters: proportional gain ' $K$ ', integral gain ' $\mathrm{Ti}$ ' and derivate gain ' $T d$ '.

\section{PID CONTROLler TUNING IN CLOSED-LOOP}

\section{A. General procedure to calculate parameters}

Two steps are necessary to obtain the controller tuning parameters in closed loop:

- It is necessary to carry the system response to a permanent oscillation state. Then certain characteristics of the response must be measured.

- According with the information achieved from the plant response, appropriate expressions must be applied to obtain correct controller parameters for the desired specifications.

\section{B. Obtaining the response characteristics in closed-loop}

Different methods can be used to obtain the controller parameters. BIO-KSY uses a relay-feedback method proposed by Aström and Hägglud [14]. The results are very similar to those obtained by the traditional method proposed for Ziegler-Nichols [16], but with some very important advantages like:

- The system operation is far from unstable state.

- Tuning can be made at any time for any working point.

The implementation scheme of relay feedback is show in figure 3. A relay with hysteresis centered on the zero value with an amplitude $d$ and a hysteresis window of width $\mathrm{h}$ is recommended for the general method.

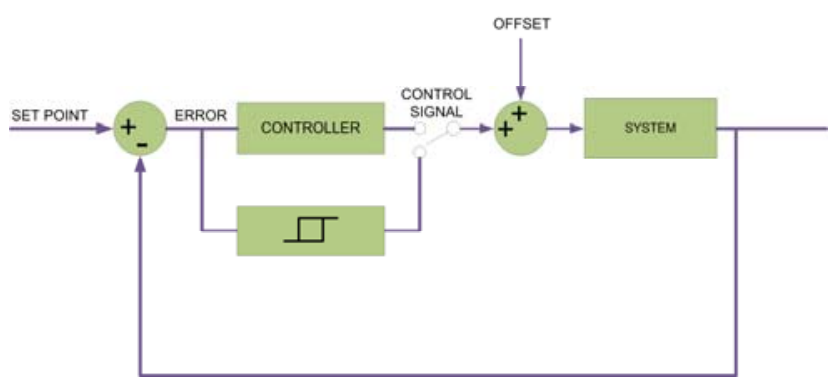

Figure 3. Typical configuration of Relay-Feedback.
The system oscillation has a period (Tc) with approximately the same value like the Ziegler-Nichols method. The critical gain $(K c)$ of the process is obtained with equation 2, where $a$ is the peak to peak value of the oscillation.

$$
K_{C}=\frac{4 d}{\pi \sqrt{a^{2}-h^{2}}}
$$

\section{Obtaining controller parameters with formulas}

With Tc and Kc characteristics of the system obtained on the previous step, controller parameters are obtained. Many expressions have been developed by different authors [14, 16-19] with the aim to achieve, among others:

- Improve a particular specification of the system controlled response.

- Make system robust to a particular criteria (Load Disturbance or Set Point Criteria)

There are several studies where different expressions were developed. Even control equipment manufacturers developed their own expressions according with their products line.

In this study four methods have been taken into account: Ziegler-Nichols, Ziegler-Nichols some overshoot, ZieglerNichols without overshoot and Tyreus-Luyben [16-19]. All of them are for Load Disturbance rejection criteria.

TABLE I. EXPRESSIONS OF CONTROLLER PARAMETERS

\begin{tabular}{|l|c|c|c|c|}
\hline & Kp & Ti & Td & Appl. Range \\
\hline Z-N & $0.6 \times \mathrm{Kc}$ & $0.5 \times \mathrm{Tc}$ & $0.125 \times \mathrm{Tc}$ & $2<\mathrm{k} \cdot \mathrm{Kc}<20$ \\
\hline Z-N Some Ov. & $0.33 \times \mathrm{Kc}$ & Tc $/ 2$ & Tc $/ 3$ & $2<\mathrm{k} \cdot \mathrm{Kc}<20$ \\
\hline Z-N Whitout Ov & $0.2 \times \mathrm{Kc}$ & Tc & Tc $/ 3$ & $2<\mathrm{k} \cdot \mathrm{Kc}<20$ \\
\hline Tyreus-Luyben & $0.45 \times \mathrm{Kc}$ & $2.2 \times \mathrm{Tc}$ & Tc $/ 6.3$ & $2<\mathrm{k} \cdot \mathrm{Kc}<20$ \\
\hline
\end{tabular}

\section{BIO-KSY}

BIO-KSY consists on a rigorous and explicit description of the domain knowledge of the controller. It is a fusion between rules and bio-inspired techniques. The conceptual general diagram of the novel bio-knowledge simplified model is shown in figure 4.

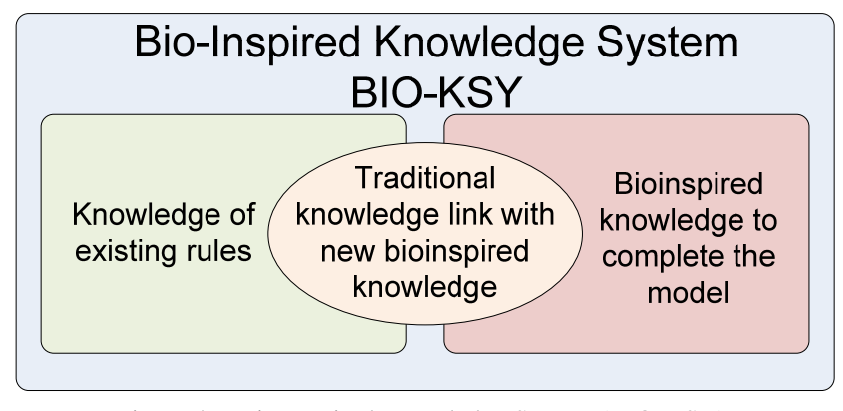

Figure 4. Bio-Inspired Knowledge System (BIO-KSY).

As shown in figure 4 the general diagram of BIO-KSY is divided in three different blocks: 
- Knowledge of existing rules. The aim of this block is to organize different rules for application ranges, authors expressions, criterions, and so on.

- Traditional knowledge link with new bioinspired knowledge. This part is the link between the other two. With it, information will be interpreted by the third block.

- Bioinspired knowledge to complete the model. This part of the model selects the most appropriate expressions to obtain the controller tuning parameters.

In general terms, the BIO-KSY will select the best tuning parameters, according with the system and the desired specifications of operation.

In the next two subsections we present the bioknowledge simplified model. The first one shows the knowledge representation corresponding of the two first blocks explained before. The second one gives details of the third block 'Bioinspired knowledge to complete the model'.

\section{A. First part of the Knowledge representation}

As a result of the different methodology of PID controller tuning in closed-loop, a flow-chart is obtained, shown in figure 5. Lots of PID tuning rules in open loop have been considered to create this diagram, with the aim to achieve a generalized knowledge of the field. In the next paragraphs the flowchart is explained in more details.

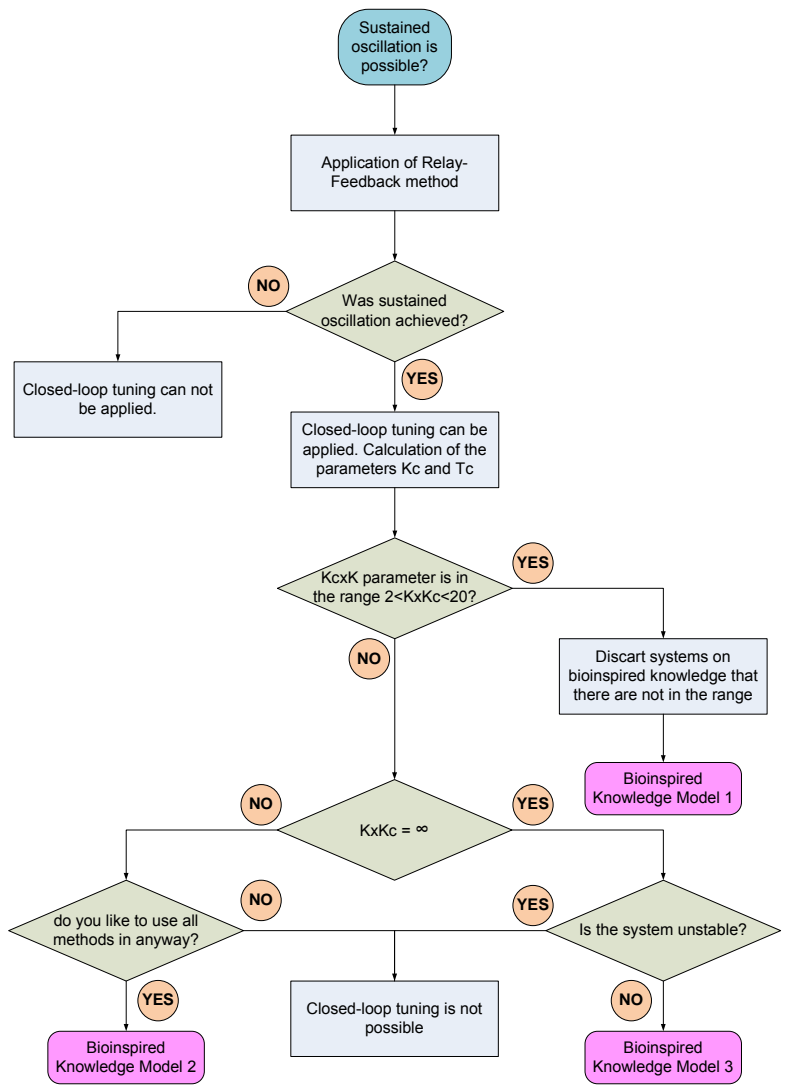

Figure 5. Flowchart corresponding with the first part of knowledge representation.
PID tuning in closed loop is only possible, if the plant engineer can put the system operation in sustained oscillation. For this reason the flowchart starts with this premise. As it has been explained in section IV there are several techniques to perform PID controller tuning in closed loop, the Relay-Feedback method, being the most robust, with which it is possible to achieve better results. It is then necessary to find out if the system can achieve the sustained oscillation. If it is not possible, then we can not apply tuning in closed loop. In other case, with the system in this state it is necessary to calculate Tc and Kc parameters.

With Tc and Kc values it is possible to obtain the K.Kc indicator, where $K$ is the gain of the process. This indicator, among others, will define the best expressions to achieve the desired operation as results of the system.

The next step in the flowchart is to determine if K.Kc indicator value is in the range of $2<\mathrm{K} . \mathrm{Kc}<20$. If it is within this range, then in order to create BIO-KSY it is required to discard all the systems that aren't within this range [14]. With the conditions met it is possible to use the bioinspired knowledge model 1.

If K.Kc indicator is out of range, the model must be able to know if the value is infinite or not. Then it is possible to follow the flowchart in two ways:

- When K.Kc is not infinite, the operator must decide if he wants to use all the expressions anyway. If he does not want to use all the expressions, the closed loop tuning is not applicable for the contemplated expressions. In other case it is possible to use the bioinspired knowledge model 2.

- When K.Kc is infinite, the operator must find out if the system is unstable. If it is unstable closed loop tuning is not applicable. This means that the system has an integrator in its transfer function. Then it is possible to use the bio knowledge model 3.

\section{B. Bioinspired Knowledge to complete the model}

As shown in figure 5, with the rules set organization, there are three blocks corresponding to the bioinspired knowledge models (1, 2 and 3). Three techniques have been applied to create these blocks: rules without machine learning, tree decision and artificial neural networks. In the next paragraphs the formulation of the model is described.

1) Model input: As can be seen in the flowchart of figure 5 and then in its description, there is a very important indicator along with it, K.Kc. It defines for instance the application range of expressions. In all bioinspired knowledge models created, K.Kc has been the input that defines the system type. Then K.Kc defines the system dynamics and consequently its controllability.

2) Dataset for model creation: As in other studies it is necessary to take representative systems with the objetive to generalize the model as much as we can. For this reason the study begins with the work [20]. This research includes a list of very representative systems, and all real systems have a similar behavior to one of them. Table II shows a list of systems included in [20] where its possible to obtain 
K.Kc. In total, the work has 32 systems where it is possible to obtain the value of K.Kc. Systems have been ordered for K.Kc value from high to low.

TABLE II. VALUES FOR THE K.KC INDICATOR IN REPRESENTATIVE SYSTEMS $(\mathrm{G} 1, \mathrm{C} 6, \ldots, \mathrm{F})$

\begin{tabular}{|c|c|c|c|c|c|}
\hline & System & K.Kc & & System & K.Kc \\
\hline 1 & G1 & 0.44 & 17 & A2 & 4 \\
\hline 2 & C6 & 0.5 & 18 & B4 & 4 \\
\hline 3 & C5 & 1.1429 & 19 & C2 & 5 \\
\hline 4 & E3 & 1.5377 & 20 & G3 & 5.24 \\
\hline 5 & E2 & 1.6422 & 21 & C1 & 6.1585 \\
\hline 6 & D3 & 1.7071 & 22 & B3 & 6.7510 \\
\hline 7 & D2 & 1.7972 & 23 & A1 & 8.0011 \\
\hline 8 & G2 & 1.8812 & 24 & D6 & 8.8672 \\
\hline 9 & A3 & 1.884 & 25 & E5 & 9.7963 \\
\hline 10 & D1 & 1.9052 & 26 & G4 & 11.2414 \\
\hline 11 & E1 & 1.9052 & 27 & E6 & 16.818 \\
\hline 12 & E4 & 1.9317 & 28 & D7 & 17.5578 \\
\hline 13 & C4 & 2 & 29 & B2 & 30.2375 \\
\hline 14 & D4 & 2 & 30 & E7 & 35.1592 \\
\hline 15 & C3 & 3.2 & 31 & B1 & 110.1 \\
\hline 16 & D5 & 3.8891 & 32 & F & 167.7135 \\
\hline
\end{tabular}

As shown in table II, the K.Kc value of the firsts systems have a very close values; however the lasts systems have widely separated values. For this reason, many systems have been created to solve the problem. For it, delay time is added to the 1th and 2nd order systems with Pade approximation [14]. Thus, we achieve a difference in the K.Kc values between consecutive systems, to be less than the unit. Finally there are 1704 systems to obtain the model. Systems obtained are balanced by controller parameters expressions.

3) System specifications for each expression: Each system is tuning with the four expresions contemplated in table I. Then four specifications are tested in all cases for step input: response time ( $T r)$, settling time (Ts), overshoot $(O v)$ and peak time $(T p)$. As a result, it is possible to obtain the expression that gives the best specification value. Table III shows examples of some systems of table II with the best specifications values for step input. The expression used to achieve this result is specified into brackets, whose legend is: Z\&N (Ziegler-Nichols), Z\&N wOv (Ziegler-Nichols Whitout Overshoot), Z\&N sOv(Ziegler-Nichols some Overshoot), T\&L (Tyreus-Luyven).

4) Classification techniques analyzed to complete the model: Three techniques have been taken into account to complete the model:

- Fisher's Linear Discriminant Analysis (FLDA): Linear Discriminat Analisys has been proposed in [21, 22]. This method overcomes other techniques with Fisher's Discriminant Criterion. It is important to highlight, that good results can be obtained with this method in too many cases, where linear classification is possible.

- Decision tree using the J48 learning algorithm: Other classification method contemplated in this research is the decision tree [23-25]. The decision tree approach is one of the most common approaches in automatic learning and decision making. The true purpose of these decision trees is to classify the data in different groups, according to the dependent variable [23]. The decision trees have been obtained by using the J48 algorithm [23, 26, 27]. The J48 algorithm was chosen because of its better performance in most circumstances [27].

- Artificial Neural Networks (ANN), Multilayer Perceptron (MLP): A multilayer perceptron is a feed forward artificial neural network [28]. It is one of the most typical ANN due to its robustness and relatively simple structure [28]. However the ANN architecture must be well selected to achieve good results. As will be seen, ANN-MLP is more difficult to apply than the other two techniques, but through it the best results were obtained.

There are many software capable of implementing these techniques like WEKA, Rapid Miner, Matlab ${ }^{\odot}$ toolbox, etc. Linear Discriminant Analysis and Multilayer Perceptron have been realized in Matlab ${ }^{\odot}$ with statistics and ANN toolboxes respectively. The J48 decision tree has been created in WEKA software. Control Toolbox of Matlab ${ }^{\odot}$ has been used for the test and the validation of results on a real plant.

TABLE III. BEST SPECIFICATIONS VALUES WITH TUNING EXPRESSIONS EXAMPLES

\begin{tabular}{|c|c|c|}
\hline System & $\operatorname{Tr}$ & Ts \\
\hline G1 & $24,45 \quad(\mathrm{Z} \& N)$ & $48,18 \quad(\mathrm{Z} \& N)$ \\
\hline C6 & $0,44(\mathrm{Z} \& \mathrm{~N}$ wOv) & $48,95 \quad(\mathrm{Z} \& N)$ \\
\hline $\mathrm{C5}$ & $7,92 \quad(\mathrm{Z} \& N)$ & $19,03 \quad(\mathrm{Z} \& N)$ \\
\hline D2 & $0,77 \quad(\mathrm{Z} \& N)$ & $5,58 \quad(\mathrm{Z} \& N)$ \\
\hline G2 & $1,05 \quad(\mathrm{Z} \& N)$ & 9,29 (Z\&N sOv) \\
\hline A3 & $4,01 \quad(\mathrm{Z} \& N)$ & $32,75 \quad(\mathrm{Z} \& N)$ \\
\hline E4 & $0,85 \quad(\mathrm{Z} \& N)$ & $7,56 \quad(\mathrm{Z} \& N)$ \\
\hline B2 & $0,12 \quad(\mathrm{Z} \& \mathrm{~N})$ & $0,9 \quad(\mathrm{~T} \& \mathrm{~L})$ \\
\hline E7 & $2,05 \quad(\mathrm{Z} \& \mathrm{~N})$ & 19,75 (T\&L) \\
\hline B1 & $0,04 \quad(\mathrm{Z} \& N)$ & $0,33 \quad(T \& L)$ \\
\hline $\mathrm{F}$ & $0,13 \quad(\mathrm{Z} \& N)$ & $1,29 \quad$ (T\&L) \\
\hline System & $\mathrm{Ov}$ & $\mathrm{Tp}$ \\
\hline G1 & 0 (Z\&N wOv) & $131,21 \quad(\mathrm{Z} \& N)$ \\
\hline C6 & 0 (Z\&N wOv) & 2,81 (Z\&N sOv) \\
\hline C5 & 0 (Z\&N wOv) & $110(\mathrm{Z \& N})$ \\
\hline D2 & 0 (Z\&N wOv) & $2,04 \quad(\mathrm{Z} \& N)$ \\
\hline G2 & 0 (Z\&N wOv) & $4,71 \quad(\mathrm{Z} \& N)$ \\
\hline A3 & 0 (Z\&N wOv) & 9,75 (Z\&N) \\
\hline E4 & 0 (Z\&N wOv) & $2,25 \quad(\mathrm{Z} \& N)$ \\
\hline $\mathrm{B} 2$ & 10,062 (Z\&N wOv) & $0,34 \quad(\mathrm{Z} \& N)$ \\
\hline E7 & $14,3172(\mathrm{Z} \& \mathrm{~N}$ wOv) & $6,34 \quad(\mathrm{Z} \& N)$ \\
\hline B1 & 20,9154 (Z\&N wOv) & $0,12 \quad(\mathrm{Z} \& \mathrm{~N})$ \\
\hline $\mathrm{F}$ & 13,2937 (Z\&N wOv) & $0,36 \quad(\mathrm{Z} \& N)$ \\
\hline
\end{tabular}

5) Classification results: For each specification (response time, settling time, overshoot and peak time) a model has been created. Each of the sets of expressions would be a class: 
- Class A: Z-N.

- $\quad$ Class B: Z-N Some Ov.

- Class C: Z-N Whitout Ov

- Class D: Tyreus-Luyben

As a method of performance measurements, five parameters have been used: Sensitivity (SE), Specificity (SPC), Positive Prediction Value (PPV), Negative Prediction Value (NPV) and Accuracy (ACC) (see equations from 3 to 7 respectively).

$$
\begin{aligned}
& S E=\frac{T P}{(T P+F N)} \\
& S P C=\frac{T N}{(F P+T N)} \\
& P P V=\frac{T P}{(T P+F P)} \\
& N P V=\frac{T N}{(T N+F N)} \\
& A C C=\frac{T P+T N}{(T P+T N+F P+F N)}
\end{aligned}
$$

Where TP is the number of True Positive, TN is the number of True Negative, FN is the number of False Negative and FP is the number of False Positive.

In all cases k-fold cross-validation has been used to split the dataset in a reasonable value that obtains good results. The $\mathrm{k}$ value is 10 for all models. For the first classification method, the discriminant analysis is of linear type. For the decision tree classification the algorithm chosen is J48 whose configuration parameters' values are: for the confidence threshold for pruning is 0.25 and for the minimum number of instances per leaf is 2 . In the case of MLP, tests have been made with 2 and 3 hidden layers, with best results for the second value. The number of neurons in hidden layer is within the range of 10-15. The activation functions tested in the hidden layer are: log sigmoid and tangent sigmoid. Best results were achieved with the tangent function type. The activation function of the output layer is the log sigmoid type. Winner Take All (WTA) technique is used to obtain the class provided by the MLP output.

Table IV shows the percentage of correct classification using the techniques mentioned before for the three Knowledge Models.

As can be seen in table IV for the knowledge model 1 548 systems, has been used, balanced by expressions type, however for the other two models (knowledge model 2 and 3) all systems have been taken into account. The difference between the number of systems of knowledge model 1 and Knowledge models 2 and 3 are due to that model 1 is created only with systems where the parameter K.Kc is in the range of $2<\mathrm{K} . \mathrm{Kc}<20$.

For all the cases contemplated in table IV, the best configuration of the used technique has been selected. For it the confusion matrix has been created in each case. As an example it is shown in tables V, VI and VII where Response Time (Tr) is tested for the following techniques: Linear

\begin{tabular}{|c|c|c|c|c|}
\hline \multicolumn{5}{|c|}{ Response Time (Tr) } \\
\hline \multirow{2}{*}{ Model data } & \multirow{2}{*}{$\begin{array}{c}\text { Training } \\
\text { data }\end{array}$} & \multicolumn{3}{|c|}{ Classification method } \\
\hline & & LDA & $\mathrm{J} 48$ & MLP \\
\hline Knowledge model 1 & 548 & 74 & 86 & 95 \\
\hline Knowledge model 2 & 1704 & 76 & 81 & 93 \\
\hline Knowledge model 3 & 1704 & 75 & 78 & 94 \\
\hline \multicolumn{5}{|c|}{ Settling Time (Ts) } \\
\hline \multirow{2}{*}{ Model data } & \multirow{2}{*}{$\begin{array}{c}\text { Training } \\
\text { data }\end{array}$} & \multicolumn{3}{|c|}{ Classification method } \\
\hline & & LDA & $\mathrm{J} 48$ & MLP \\
\hline Knowledge model 1 & 548 & 73 & 84 & 96 \\
\hline Knowledge model 2 & 1704 & 70 & 87 & 94 \\
\hline Knowledge model 3 & 1704 & 71 & 83 & 94 \\
\hline \multicolumn{5}{|c|}{ Overshoot (Ov) } \\
\hline \multirow{2}{*}{ Model data } & \multirow{2}{*}{$\begin{array}{c}\text { Training } \\
\text { data }\end{array}$} & \multicolumn{3}{|c|}{ Classification method } \\
\hline & & LDA & $\mathrm{J} 48$ & MLP \\
\hline Knowledge model 1 & 548 & 71 & 82 & 97 \\
\hline Knowledge model 2 & 1704 & 74 & 83 & 92 \\
\hline Knowledge model 3 & 1704 & 77 & 86 & 95 \\
\hline \multicolumn{5}{|c|}{ Peak Time (Tp) } \\
\hline \multirow{2}{*}{ Model data } & \multirow{2}{*}{$\begin{array}{c}\text { Training } \\
\text { data }\end{array}$} & \multicolumn{3}{|c|}{ Classification method } \\
\hline & & LDA & $\mathrm{J} 48$ & MLP \\
\hline Knowledge model 1 & 548 & 75 & 83 & 89 \\
\hline Knowledge model 2 & 1704 & 69 & 79 & 91 \\
\hline Knowledge model 3 & 1704 & 74 & 80 & 93 \\
\hline
\end{tabular}
Discriminant Analysis, J48 Decision Tree and Multi Layer Perceptron respectively.

TABLE IV. PERCENTAGE OF CORRECT CLASSIFICATION FOR TRHEE KNOWLEDGE MODELS

TABLE V. CONFUSION MATRIX FOR CLASSIFICATION RATE OF OF RESPONSE TIME OF KNOWLEDGE MODEL 1 USING LDA

\begin{tabular}{|c|c|c|c|c|}
\hline & \multicolumn{4}{|c|}{ Method chosen by model } \\
\hline Desired Method & A & B & C & D \\
\hline A & $\mathbf{2 1 0}$ & 72 & 76 & 68 \\
\hline B & 85 & $\mathbf{2 2 8}$ & 64 & 49 \\
\hline C & 74 & 89 & $\mathbf{1 9 8}$ & 65 \\
\hline D & 77 & 54 & 92 & $\mathbf{2 0 3}$ \\
\hline TP & 210 & 228 & 198 & 203 \\
\hline TN & 1042 & 1063 & 1046 & 1096 \\
\hline FP & 236 & 215 & 232 & 182 \\
\hline FN & 216 & 198 & 228 & 223 \\
\hline SE & 0,493 & 0,535 & 0,465 & 0,477 \\
\hline SPC & 0,815 & 0,832 & 0,818 & 0,858 \\
\hline PPV & 0,471 & 0,515 & 0,460 & 0,527 \\
\hline NPV & 0,828 & 0,843 & 0,821 & 0,831 \\
\hline ACC & 0,735 & 0,758 & 0,730 & 0,762 \\
\hline
\end{tabular}


TABLE VI CONFUSION MATRIX FOR CLASSIFICATION RATE OF OF RESPONSE TIME OF KNOWLEDGE MODEL 1 USING J48 DECISION TREE

\begin{tabular}{|c|c|c|c|c|}
\hline & \multicolumn{5}{|c|}{ Method chosen by model } \\
\hline Desired Method & A & B & C & D \\
\hline A & $\mathbf{3 2 2}$ & 42 & 38 & 24 \\
\hline B & 28 & $\mathbf{3 3 9}$ & 36 & 23 \\
\hline C & 46 & 27 & $\mathbf{3 0 2}$ & 51 \\
\hline D & 41 & 49 & 42 & $\mathbf{2 9 4}$ \\
\hline TP & 322 & 339 & 302 & 294 \\
\hline TN & 1163 & 1160 & 1162 & 1180 \\
\hline FP & 115 & 118 & 116 & 98 \\
\hline FN & 104 & 87 & 124 & 132 \\
\hline SE & 0,756 & 0,796 & 0,709 & 0,690 \\
\hline SPC & 0,910 & 0,908 & 0,909 & 0,923 \\
\hline PPV & 0,737 & 0,742 & 0,722 & 0,750 \\
\hline NPV & 0,918 & 0,930 & 0,904 & 0,899 \\
\hline ACC & 0,871 & 0,880 & 0,859 & 0,865 \\
\hline \multicolumn{5}{|l}{}
\end{tabular}

TABLE VII. CONFUSION MATRIX FOR CLASSIFICATION RATE OF OF RESPONSE TIME OF KNOWLEDGE MODEL 1 USING MLP

\begin{tabular}{|c|c|c|c|c|}
\hline & \multicolumn{4}{|c|}{ Method chosen by model } \\
\hline Desired Method & A & B & C & D \\
\hline A & $\mathbf{3 9 9}$ & 9 & 15 & 3 \\
\hline B & 9 & $\mathbf{4 0 1}$ & 6 & 10 \\
\hline C & 10 & 18 & $\mathbf{3 8 3}$ & 15 \\
\hline D & 17 & 12 & 19 & $\mathbf{3 7 8}$ \\
\hline TP & 399 & 401 & 383 & 378 \\
\hline TN & 1242 & 1239 & 1238 & 1250 \\
\hline FP & 36 & 39 & 40 & 28 \\
\hline FN & 27 & 25 & 43 & 48 \\
\hline SE & 0,937 & 0,941 & 0,899 & 0,887 \\
\hline SPC & 0,972 & 0,969 & 0,969 & 0,978 \\
\hline PPV & 0,917 & 0,911 & 0,905 & 0,931 \\
\hline NPV & 0,979 & 0,980 & 0,966 & 0,963 \\
\hline ACC & 0,963 & 0,962 & 0,951 & 0,955 \\
\hline
\end{tabular}

\section{EMPIRICAL VERIFICATION ON PHYSICAL PLANT}

An empirical verification of BIO-KSY was performed on a laboratory plant (figure 6) in which the tank level is controlled by adjusting the speed of the centrifugal pump.

1) Physical description: The real plant used for these experiments consists of a tank, fed at the top by the liquid from the pump; speed of the pump will be controlled to maintain a constant water level while emptying through an output valve.

The regulator is a virtual controller that takes signals from the plant through a data acquisition card. As a set point signal, the plant receives the required water level and adjusts the speed of centrifugal pump to set the input flow into the tank.

2) Implementation of the control: The method was run in the Simulink environment. For operations at the plant, a National Instruments data acquisition card (model USB6008 12-bit $10 \mathrm{KS} / \mathrm{s}$ Multifunction I/O) was chosen. The diagram of the process is implemented in Simulink with the control Scheme shown in figure 7. Different gain blocks have been added to adapt signal levels to all operation range.

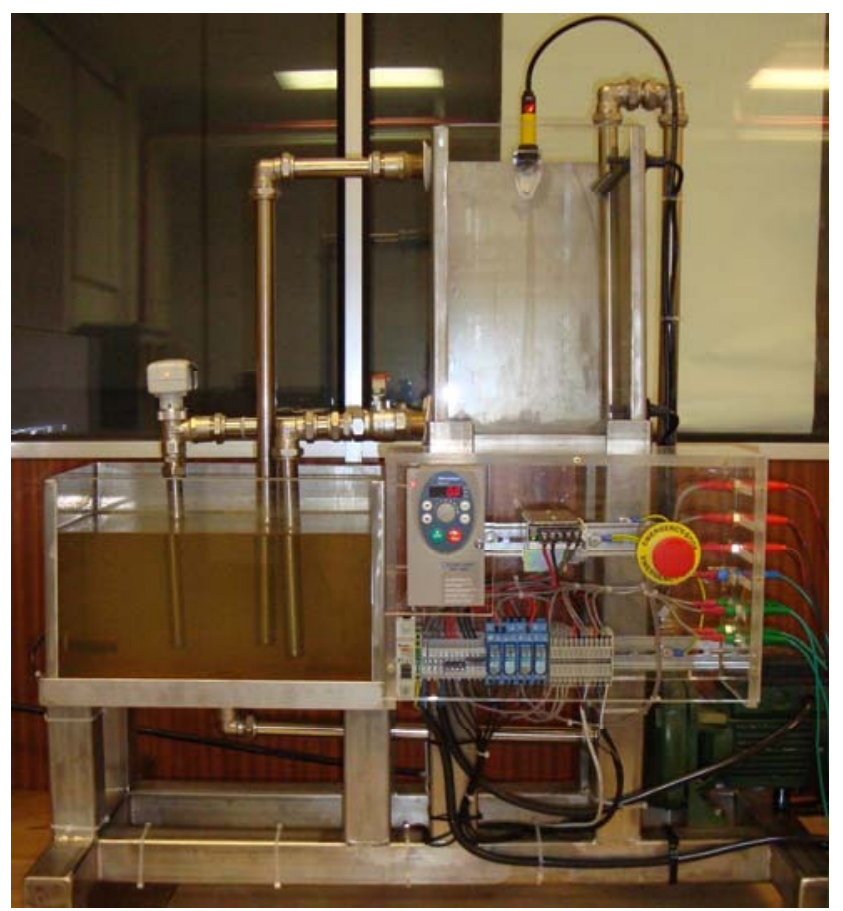

Figure 6. Photograph and scheme of the real plant.

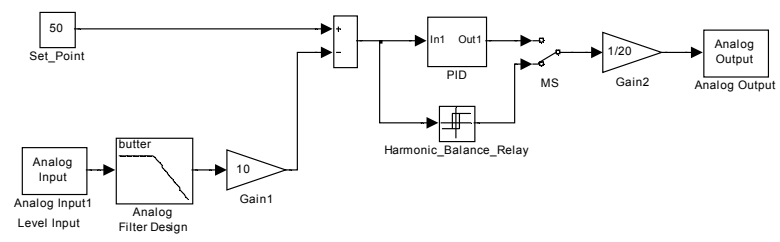

Figure 7. Control scheme implemented in Simulink.

It was necessary to add a filter block whose edge frequency is $1.5 \mathrm{rad} / \mathrm{sec}(9.5 \mathrm{~Hz})$ in order to reduce noise from the analog input. Through a switch it is possible to select either a PID control or Relay-Feedback configuration. Figure 8 shows an internal implementation of a PID block. PID controller gains (Ti, Td, and $\mathrm{K}$ ) are programmed manually.

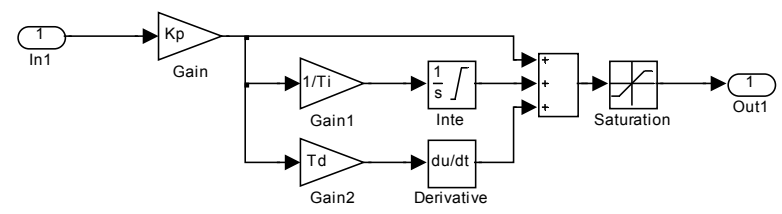

Figure 8. PID block internal scheme. 
3) Practical behavior of BIO-KSY application onto a physical plant: Some tests have been made on physical described system before, with the aim to check the behavior of the novel bio-knowledge model.

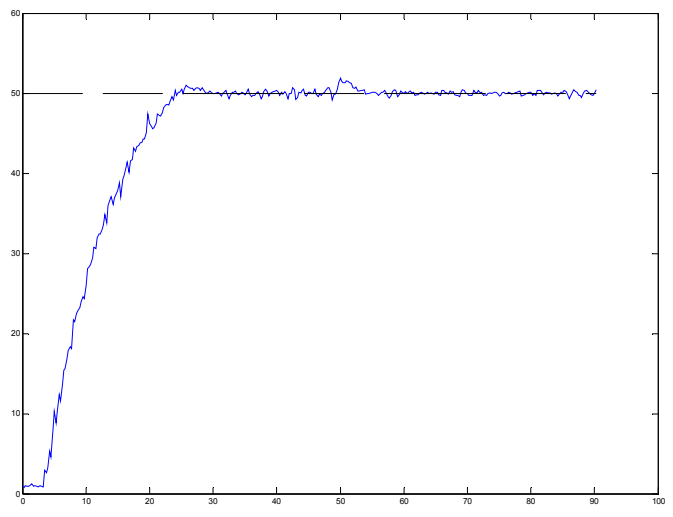

Figure 9. System response with method from Bioknowledge model.

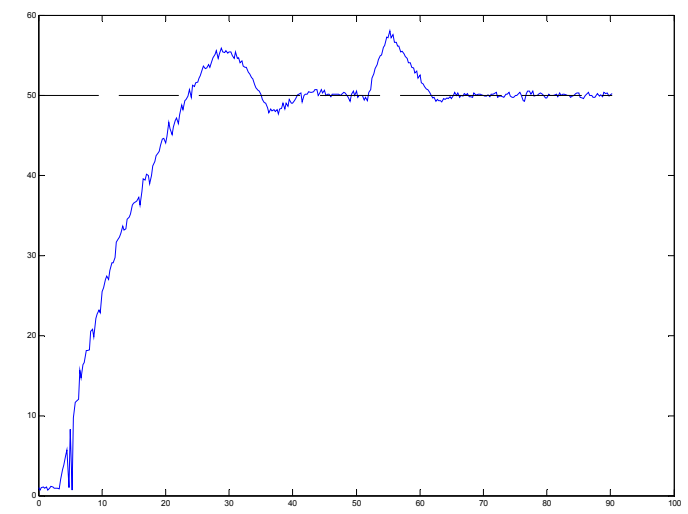

Figure 10. System response with Ziegler-Nichols method in closed-loop.

The first step was to apply relay-feedback method on the physical system. For it, the scheme of figure 7 is closed through the relay block, and it is set to operation. When oscillation is in steady state, Tc and a (to obtain Kc) parameters are measured $(\mathrm{Tc}=16.63 \mathrm{~s}$ and $\mathrm{a}=1.42 \rightarrow \mathrm{Kc}=$ 12.53)). Then K.Kc indicator is obtained in order to apply it as an input on the Bioknowledge model $(\mathrm{K} . \mathrm{Kc}=1.2 \times 12.53=$ 15.04). BIO-KSY is able to obtain the correct expressions for K.Kc value. With the selected expressions PID parameters $(\mathrm{K}, \mathrm{Ti}$ and $\mathrm{Td})$ are calculated and programmed within the Matlab/Simulink environment. With $\mathrm{K} . \mathrm{Kc}=15.04$, if the operator needs to improve the overshoot, for example he applies the Tyreus-Luyben expressions. The PID parameters' values using these expressions are: $\mathrm{K}=4.14, \mathrm{Ti}=8.31$ and $\mathrm{Td}$ $=5.54$. The system response with a step input for the work point of the design is shown on figure 9 . Figure 10 shows the response with a step input for a traditional Ziegler-Nichols method in closed loop. As can be seen in these figures the bioknowledge model chooses the best expressions to achieve the best overshoot between the expressions contemplated in the study. In both cases a perturbation is introduced, but the first method is more robust.

\section{CONCLUSIONS}

A novel Bioknowledge model has been presented in this study, tested with a practical application in all control loops where PID controller is used. The best advantage offered by the method is that, it assures the most appropriate selection of the expressions to obtain the PID controller parameters.

It is necessary to highlight that the novel model is easy to expand to other system types. It is only necessary then to contemplate the new systems in order to create the Bioinspired Knowledge models. The rest of the model is completely valid.

With the aim to compare and make the model more robust some techniques have been taken into consideration: Linear Discriminant Analysis, decision trees J48 and Multilayer Perceptron.

Several future research lines have been considered. Essentially two of them are the most important. Firstly it is necessary to contemplate other bio-inspired and intelligent techniques to improve the model despite the very good results that have been already obtained. The other important research line is to make tests on real industrial plants to analyze the results.

\section{ACKNOWLEDGMENT}

This research is partially supported by projects TIN201021272-C02-01 from the Spanish Ministry of Science and Innovation. The authors would also like to thank the manufacturer of components for vehicle interiors, Grupo Antolin Ingeniería, S.A. which provided support through MAGNO 2008 - 1028 - CENIT funded by the Spanish Ministry of Science and Innovation.

\section{REFERENCES}

[1] C. Hsu, G. Chen, and T. Lee, "Robust intelligent tracking control with PID-type learning algorithm," Neurocomputing, vol. 71, Dec. 2007, pp. 234-243.

[2] S. Gottwald, "Mathematical Fuzzy Control. A Survey of Some Recent Results,” Logic Journal of IGPL, vol. 13, Sep. 2005, pp. 525541.

[3] J. Zhang, J. Zhuang, H. Du, and S. Wang, "Self-organizing genetic algorithm based tuning of PID controllers," Information Sciences, vol. 179, Mar. 2009, pp. 1007-1018.

[4] Y. Juang, Y. Chang, and C. Huang, "Design of fuzzy PID controllers using modified triangular membership functions," Information Sciences, vol. 178, Mar. 2008, pp. 1325-1333.

[5] H. Liu and G. Coghill, "A model-based approach to robot fault diagnosis,” Knowledge-Based Systems, vol. 18, Aug. 2005, pp. 225233.

[6] H. Lu, J. Chang, and M. Yeh, "Design and analysis of direct-action CMAC PID controller,” Neurocomputing, vol. 70, Oct. 2007, pp. 2615-2625. 
[7] A. Sala, Á. Cuenca, and J. Salt, “A retunable PID multi-rate controller for a networked control system,” Information Sciences, vol. 179, Jun. 2009, pp. 2390-2402.

[8] R.R. Sumar, A.A.R. Coelho, and L.D.S. Coelho, "Computational intelligence approach to PID controller design using the universal model,” Information Sciences, vol. 180, Oct. 2010, pp. 3980-3991.

[9] R. Thangaraj, T.R. Chelliah, M. Pant, a Abraham, and C. Grosan, "Optimal gain tuning of PI speed controller in induction motor drives using particle swarm optimization,” Logic Journal of IGPL, vol. 19, Jul. 2010, pp. 343-356.

[10] Y. Juang, Y. Chang, and C. Huang, "Design of fuzzy PID controllers using modified triangular membership functions," Information Sciences, vol. 178, Mar. 2008, pp. 1325-1333.

[11] J. Ye, "Adaptive control of nonlinear PID-based analog neural networks for a nonholonomic mobile robot,” Neurocomputing, vol. 71, Mar. 2008, pp. 1561-1565.

[12] J.A. Romero, R. Sanchis, and P. Balaguer, "PI and PID auto-tuning procedure based on simplified single parameter optimization," Journal of Process Control, vol. 21, Jul. 2011, pp. 840-851.

[13] J. Sun, D.-hua Zhang, X. Li, J. Zhang, and D.-shun Du, "Smith Prediction Monitor AGC System Based on Fuzzy Self-Tuning PID Control,” Journal of Iron and Steel Research, International, vol. 17, Feb. 2010, pp. 22-26.

[14] K.J. Astrom and T. Hagglund, “Advanced PID Control”. Pearson Education. Madrid. 2009

[15] Y.L. Feng and K.C. Tan, "Process identification and PID Control". John Wiley\&Sons. USA. 2009

[16] A. Visioli, "Practical PID Control”. Springer-Verlag, London. 2010

[17] M.A. Johnson, M.H. Moradi, "PID Control: New identification and Design methods" Springer-Verlag, London. 2010
[18] H. Seki, T. Shigemasa, Retuning oscillatory PID control loops based on plant operation data, J. Process Control vol. 20. 2010. pp. 217227.

[19] B.D. Tyreus, W.L. Luyben, "Tuning PI controllers for integrator/dead time processes" Industrial Engineering Chemistry Research, 1992, 31(11), 2625-2628.

[20] K.J. Astrom, T. Hagglund, "Benchmark Systems for PID Control" Preprints FAC Workshop on Digital Control. Past, present and future of PID Control Tarrasa 2000. pp. 181-182

[21] P.N. Belhumeur, J.P. Hespanha, and D.J. Kriegman, "Eigenfaces vs. Fisherfaces: recognition using class specific linear projection,” IEEE Transactions on Pattern Analysis and Machine Intelligence, vol. 19, Jul. 1997, pp. 711-720.

[22] D.L. Swets and J.J. Weng, "Using Discriminant Eigenfeatures for Image Retrieval” IEEE Trans. Pattern Analysis and Machine Intelligence, vol. 18, no. 8, pp. 831-836, Aug. 1996.

[23] O. Parr "Data Mining Cookbook. Modeling Data for Marketing, Risk, and Customer Relationship Management”. John Wiley \& Sons, Inc. New York. 2001

[24] R.O. Duda, P.E. Hart, D.G. Stork "Pattern Classification”. John Wiley \& Sons, Inc. Canada 2001

[25] T. M. Mitchell “Machine Learning”. McGraw-hill, New York 1997

[26] E. Frank, I. Witten "Data Mining: Practical Machine Learning Tools and Techniques”. Second Edition. Morgan Kaufmann. 2005

[27] L. Rokach, O. Maimon, "Data Mining with Decision Trees: Theory and Applications". World Scientific Publishing. USA. 2008.

[28] E. Alpaydin, "Introduction to Machine Learning”, The MIT Press. Oxford, 2009. 